\title{
Dynamics of Vibratory Bowl Feeders
}

\author{
J.M. Selig \\ Faculty of Business, Computing and Info. Management. \\ London South Bank University, \\ London SE1 OAA, U.K. \\ seligjm@lsbu.ac.uk
}

\begin{abstract}
In this work we construct a simple dynamical model for vibratory bowl feeders. The symmetrical arrangement of the springs supporting the bowl allow us to predict a simple structure for the stiffness matrix of the system. The cylindrical symmetry of the bowl itself then means that the linearized rigid body dynamics of the system can be simplified to a 2-dimensional system. The solutions to this system are elliptical motions of the bowl, vibrating about the symmetry axis and along it at the same time. We are able to find a condition for the system to be at resonance. There is some debate about how the parts move up the helical track inside the bowl. We are able to show that one alternative, a "slipstick" motion, is unlikely.
\end{abstract}

Index Terms - Vibratory bowl feeders, stiffness matrix, screw theory.

\section{INTRODUCTION}

Vibratory bowl feeders are common devices used to orient and feed components to be assembled, see Fig. 1. Despite the ubiquity of these devices in manufacturing most recent academic interest has focused on two areas: The design of traps or fences which ensure that the fed parts are in a particular orientation, (see for example [10]), and the mechanics of motion of parts on vibrating plates and tracks, (see [3]).

In this work we look at the dynamics of the device itself with a view to optimising its design. This involves modelling the dynamics of the vibrating bowl. The aim is to understand how the size and placement of the leaf springs affects the motion of the bowl and how this in turn determines the motion of the parts to be fed. Early work, such as [1] and [4] studied linear vibratory feeders but concentrated on the motion of the fed parts. In [6] the frequencies and shape of free vibrational modes were determined and verified experimentally. Later, a simplified model for the dynamics of the bowl was included in [5]. Most recently, a force analysis of these devices was developed in [9].

Here we are able to give a fairly complete description of the motion of the bowl. Employing screw theory we are able to exploit the symmetry of the device, the symmetrical arrangement of the springs and the cylindrical symmetry of the bowl. This quickly leads to a simplification of the dynamics to a 2 degree-of-freedom system. We look in some detail at the stiffness matrix associated with the leaf springs. Finally we are able to show that the fed parts are

\author{
Jian S. Dai \\ Dept. Mechanical Engineering. \\ King's College, Strand, \\ London WC2R 2LS, U.K. \\ jian.dai@kcl.ac.uk
}

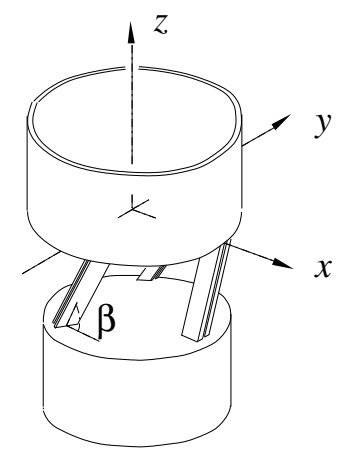

Fig. 1. A typical vibratory bowl feeder.

likely to undergo a bouncing motion as discussed in [3] rather than a "stick-slip" motion.

Our second aim: to understand how the motion of the parts is affected by the motion of the bowl, has not been addressed here. This paper should be viewed as a report on work in progress.

We begin with a brief review of some of the ideas and notation used in the rest of this work.

\section{SCREWS AND WRENCHES}

A twist or screw is an element of the Lie algebra to the group of rigid body motions $S E(3)$. These sixdimensional vectors are usually used to represent velocities of rigid bodies but here we will use them to denote small displacements,

$$
\mathbf{s}=\left(\begin{array}{c}
\boldsymbol{\delta} \boldsymbol{\theta} \\
\boldsymbol{\delta} \mathbf{p}
\end{array}\right)=\left(\begin{array}{l}
\delta \theta_{x} \\
\delta \theta_{y} \\
\delta \theta_{z} \\
\delta p_{x} \\
\delta p_{y} \\
\delta p_{z}
\end{array}\right)
$$

Suppose that $\mathbf{r}$ is a point in space, then the small displacement in the position of $\mathbf{r}$ represented by the screw $\mathbf{s}$ will be given by

$$
\delta \mathrm{r}=\delta \theta \times \mathbf{r}+\delta \mathrm{p}
$$

Under a rigid body motion, screws transform according to the adjoint representation of $S E(3)$. A group element 
in this representation can be conveniently written in partitioned form as,

$$
H=\left(\begin{array}{cc}
R & 0 \\
T R & R
\end{array}\right)
$$

where $R$ is a $3 \times 3$ rotation matrix and $T$ is a $3 \times 3$ antisymmetric matrix corresponding to a translation. That is, $T \mathbf{x}=\mathbf{t} \times \mathbf{x}$ for any vector $\mathbf{x}$, where $\mathbf{t}$ is the translation vector. The active transformation of a screw $\mathbf{s}$ by such a rigid motion is given by the matrix product, $\mathbf{s}^{\prime}=H \mathbf{s}$.

The force and torque acting on a rigid body is written as a single six-dimensional vector called a wrench,

$$
\mathcal{W}=\left(\begin{array}{c}
M_{x} \\
M_{y} \\
M_{z} \\
F_{x} \\
F_{y} \\
F_{z}
\end{array}\right),
$$

where $F_{i}$ are the components of the force and $M_{i}$ the components of the moment acting on the body. Under a rigid body motion these vectors transform according to the coadjoint representation of $S E(3)$. So an active transformation specified by a rotation $R$ and a translation $T$ will transform a wrench $\mathcal{W}$ according to,

$$
\mathcal{W}^{\prime}=H^{-T} \mathcal{W}
$$

where we have used the abbreviation $H^{-T}$ for $\left(H^{-1}\right)^{T}$.

It is simple to check that the work done by a wrench $\mathcal{W}$ over a displacement $\mathbf{s}$ is given by,

$$
\text { Work }=\mathcal{W}^{T} \mathbf{s} .
$$

It is also easy to see that the work doesn't change if the wrench and screw are both transformed by the same rigid motion.

A generalised version of Hooke's law can be written

$$
\mathcal{W}=K \mathbf{s}
$$

where $K$ is the $6 \times 6$ stiffness matrix of the system. If we subject the system to a rigid body motion $H$ then the stiffness matrix will transform according to

$$
K^{\prime}=H^{-T} K H^{-1} .
$$

Notice that this transformation law ensures that the elastic energy in the system, given by $(1 / 2) \mathbf{s}^{T} K \mathbf{s}$, is invariant under rigid body motions.

More details on this Lie algebraic approach to mechanics can be found in [7] and particular details about stiffness matrices in [8].

\section{SyMmetry OF THE STIFFnESS MATRIX}

In general, a vibratory bowl feeder consists of a cylindrical bowl connected to a heavy base by three or four leaf springs. These springs are placed symmetrically about the axis of the bowl but may be inclined with respect to the axis.

The symmetrical placement of the springs means that the stiffness matrix of the whole system has a rather simple form. Suppose that the stiffness matrix of a single spring is given by $K_{0}$, for a bowl with 3 springs the stiffness matrix of the system will be given by,

$$
K=K_{0}+H^{-T} K_{0} H^{-1}+\left(H^{-T}\right)^{2} K_{0}\left(H^{-1}\right)^{2} .
$$

Here $H$ represents a rotation of $2 \pi / 3$ about the axis of the bowl. If we take the axis of the bowl to be the $z$-axis of the co-ordinate frame then $H$ has the form,

$$
H=\left(\begin{array}{cc}
R & 0 \\
0 & R
\end{array}\right),
$$

where the rotation matrix $R$ is given by,

$$
R=\left(\begin{array}{ccc}
\cos (2 \pi / 3) & -\sin (2 \pi / 3) & 0 \\
\sin (2 \pi / 3) & \cos (2 \pi / 3) & 0 \\
0 & 0 & 1
\end{array}\right) .
$$

Let us write the stiffness matrix in partitioned form,

$$
K=\left(\begin{array}{cc}
\Xi & \Gamma \\
\Gamma^{T} & \Upsilon
\end{array}\right)
$$

Now it is clear that the stiffness matrix will satisfy the symmetry condition

$$
H^{-T} K H^{-1}=K .
$$

with $H$ as in equation (10) above, since $H^{3}=I$. This implies that, in these coordinates the parts of stiffness matrix have the form,

$$
\begin{gathered}
\Xi=\left(\begin{array}{ccc}
\xi_{11} & 0 & 0 \\
0 & \xi_{11} & 0 \\
0 & 0 & \xi_{33}
\end{array}\right), \\
\Gamma=\left(\begin{array}{ccc}
\gamma_{11} & \gamma_{12} & 0 \\
-\gamma_{12} & \gamma_{11} & 0 \\
0 & 0 & \gamma_{33}
\end{array}\right)
\end{gathered}
$$

and

$$
\Upsilon=\left(\begin{array}{ccc}
u_{11} & 0 & 0 \\
0 & u_{11} & 0 \\
0 & 0 & u_{33}
\end{array}\right)
$$

This form doesn't really depend on the number of springs, as long as the rotation angle of the symmetry operation is not 0 or $\pi$ we get the same result. 


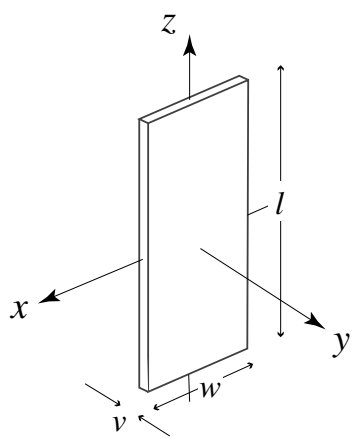

(a)

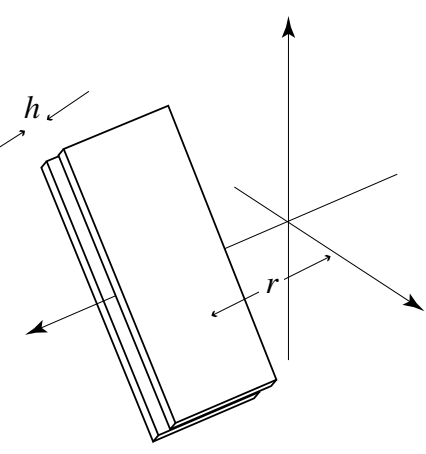

(b)

Fig. 2. Leaf springs, (a) Single leaf and (b) two leaves.

\section{LEAF SPRINGS}

Now we look more closely at the values of the non-zero components of the stiffness matrix. The stiffness matrix of a simple beam is a $6 \times 6$ diagonal matrix,

$$
K_{1}=\operatorname{diag}\left(\frac{E J_{x}}{l}, \frac{E J_{y}}{l}, \frac{G J}{l}, \frac{12 E J_{y}}{l^{3}}, \frac{12 E J_{x}}{l^{3}}, \frac{E A}{l}\right),
$$

see [11] or [2]. Here $E$ is the Young's modulus and $G$ the shear modulus of the beam material. The length of the beam is denoted by $l$ and if we assume that the beam has a rectangular cross-section with thickness $v$ and breadth $w$ then the area integral are

$$
A=v w, J_{x}=\frac{v^{3} w}{12}, J_{y}=\frac{v w^{3}}{12}, J=\frac{v w}{12}\left(v^{2}+w^{2}\right) .
$$

See Fig. 2 (a).

Leaf springs consist of several of these beams connected in parallel. To find the stiffness matrix for a pair of leaves we simply add their individual stiffness matrices. However to do this addition we must have the individual stiffness matrices in a common coordinate frame. If we assume that the separation between the leaves is $h$ then we can translate the stiffness matrix for a single leaf by $+h / 2$ and $-h / 2$ in the $y$-direction. See Fig. 2 (b). Adding the resulting stiffness matrices gives a diagonal matrix again, $K_{2}=\operatorname{diag}\left(d_{1}, d_{2}, d_{3}, d_{4}, d_{5}, d_{6}\right)$, but now the entries are,

$$
\begin{aligned}
d_{1} & =\frac{E\left(4 J_{x}-h^{2} A\right)}{2 l} \\
d_{2} & =\frac{2 E J_{y}}{l} \\
d_{3} & =\left(\frac{G J}{l}-\frac{6 h^{2} E J_{y}}{l^{3}}\right) \\
d_{4} & =\frac{24 E J_{y}}{l^{3}} \\
d_{5} & =\frac{24 E J_{x}}{l^{3}} \\
d_{6} & =\frac{2 E A}{l} .
\end{aligned}
$$

It is clear how this can be generalised for more leaves.

To get $K_{0}$ we must translate the stiffness matrix $K_{2}$ along the $x$-axis and rotate it about this axis, see Fig. 2 (b) again. We will denote the rotation angle $\beta$ here and write the distance translated as $r$. The result is simple to derive but rather lengthy to present. In the following section we argue that only a few of the entries are relevant to the dynamics. Finally to find the elements of $K$ we must transform and add as in equation (9) above. For the elements we are interested in this amounts to multiplying the elements of $K_{0}$ by 3 , we get,

$$
\begin{aligned}
& \xi_{33}= 3\left(d_{2} \sin ^{2} \beta+d_{3} \cos ^{2} \beta+\right. \\
&\left.r^{2} d_{5} \sin ^{2} \beta+r^{2} d_{6} \cos ^{2} \beta\right), \\
& \gamma_{33}=3 r\left(d_{5}-d_{6}\right) \cos \beta \sin \beta, \\
& u_{33}=3\left(d_{5} \sin ^{2} \beta+d_{6} \cos ^{2} \beta\right) .
\end{aligned}
$$

\section{LINEARIZED DYNAMICS}

We consider small motions of the feeder about its equilibrium position. The small displacement of the bowl can be described by a twist, $\mathbf{s}^{T}=\left(\theta_{x}, \theta_{y}, \theta_{z}, x, y, z\right)$, where $\theta_{x}$ is the small angle of rotation about the $x$-axis, $x$ is a small translation along the $x$-axis and so forth. The linearized dynamics of the feeder are given by,

$$
N \ddot{\mathbf{s}}+K \mathbf{s}=\mathcal{W} .
$$

Here, $\mathcal{W}$ is the driving wrench and $N$ is the $6 \times 6$ inertia matrix of the bowl.

Now the bowl of the feeder has approximately cylindrical symmetry with the symmetry axis aligned along the symmetry axis of the total stiffness matrix. Assuming that the axis of symmetry is the $z$-axis as in the previous sections, the inertia matrix will have the form,

$$
N=\left(\begin{array}{cc}
D & C \\
C^{T} & m I
\end{array}\right)
$$

where $D$ is a diagonal matrix,

$$
D=\left(\begin{array}{ccc}
m k_{1}^{2} & 0 & 0 \\
0 & m k_{1}^{2} & 0 \\
0 & 0 & m k^{2}
\end{array}\right)
$$

the mass of the bowl is $m$ here and the $k \mathrm{~s}$ are radii of gyration. Note that the cylindrical symmetry implies that 

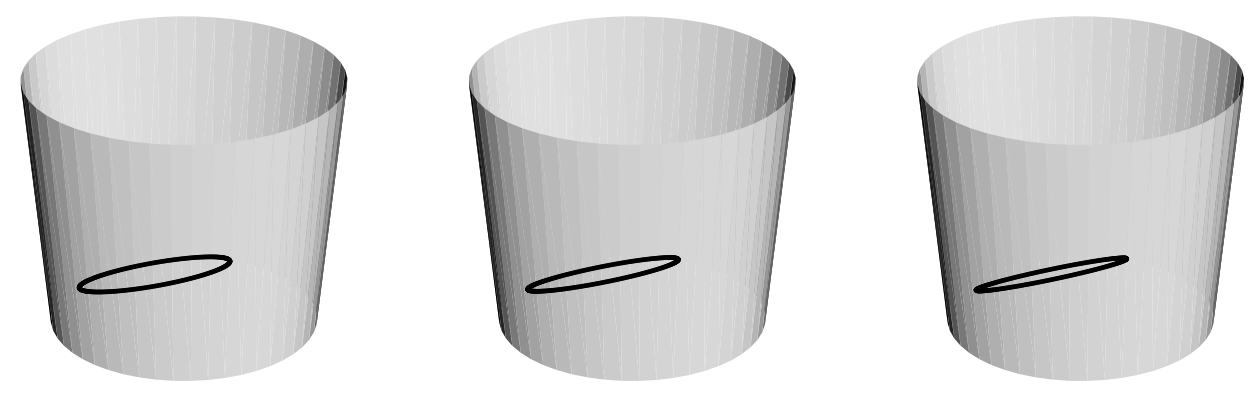

Fig. 3. The motion of a point on the bowl showing three different phase differences.

the radius of gyration about the $x$-axis $k_{1}$, is the same as the radius of gyration about the $y$-axis. The matrix $C$ gives the position of the centre of mass which must lie on the symmetry axis of course, say a distance $c_{z}$ from the origin:

$$
C=\left(\begin{array}{ccc}
0 & -m c_{z} & 0 \\
m c_{z} & 0 & 0 \\
0 & 0 & 0
\end{array}\right),
$$

finally here $I$ denotes the $3 \times 3$ identity matrix.

Normally the machine will be actuated by several strong electromagnets, these are arranged so as to produce a force in the $z$-direction and simultaneously a torque about the $z$ axis. The electromagnets are driven cyclically so that the wrench acting on the bowl can be represented as,

$$
\mathcal{W}=\left(\begin{array}{c}
0 \\
0 \\
p A \\
0 \\
0 \\
A
\end{array}\right) \cos \omega t
$$

where $p$ is the pitch of the wrench, $A$ the amplitude and $\omega$ the driving frequency of the magnets and $t$ represents time. In some designs the actuators are arranged so as to produce a pure torque $\mathcal{W}^{T}=(0,0, A, 0,0,0) \cos \omega t$, with the conventions adopted here this is a wrench with infinite pitch.

Notice that the equations de-couple. Consider the set of wrenches comprising forces along the $z$-axis together with torques about the $z$-axis, clearly the driving wrench of the machine lies in this linear system. Now we can separate the equations into two groups, 4 equations are annihilated by any wrench in the linear system. The degrees of freedom represented by these equations are not driven and hence we can assume that initial motion will be damped out after a while and hence there will be no motion here. We will not consider these degrees of freedom further. The remaining pair of equations can be written,

$$
\begin{aligned}
& \left(\begin{array}{cc}
m k^{2} & 0 \\
0 & m
\end{array}\right)\left(\begin{array}{c}
\ddot{\theta}_{z} \\
\ddot{z}
\end{array}\right)+ \\
& \quad\left(\begin{array}{ll}
\xi_{33} & \gamma_{33} \\
\gamma_{33} & u_{33}
\end{array}\right)\left(\begin{array}{c}
\theta_{z} \\
z
\end{array}\right)=\left(\begin{array}{c}
p A \\
A
\end{array}\right) \cos \omega t .
\end{aligned}
$$

From now on we will simply write $\xi$ for $\xi_{33}, \gamma$ for $\gamma_{33}$ and so forth.
In practical machines there will be damping, in line with our linearized dynamics we can include a damping matrix to provide a wrench which is a linear function of the velocity of the bowl. A reasonable assumption that is often made is that the damping matrix is proportional to the stiffness matrix, so called proportional damping,

$$
\begin{gathered}
\left(\begin{array}{cc}
m k^{2} & 0 \\
0 & m
\end{array}\right)\left(\begin{array}{c}
\ddot{\theta}_{z} \\
\ddot{z}
\end{array}\right)+\nu\left(\begin{array}{cc}
\xi & \gamma \\
\gamma & u
\end{array}\right)\left(\begin{array}{c}
\dot{\theta}_{z} \\
\dot{z}
\end{array}\right)+ \\
\left(\begin{array}{cc}
\xi & \gamma \\
\gamma & u
\end{array}\right)\left(\begin{array}{c}
\theta_{z} \\
z
\end{array}\right)=\left(\begin{array}{c}
p A \\
A
\end{array}\right) \cos \omega t .
\end{gathered}
$$

It is possible to consider other damping matrices, however if the damping is not symmetrical about the $z$-axis then the equations will not de-couple.

The steady state solutions to the above equations are sinusoids of angular frequency $\omega$ that is,

$$
\begin{aligned}
\theta_{z} & =A_{1} \cos \left(\omega t+\phi_{1}\right), \\
z & =A_{2} \cos \left(\omega t+\phi_{2}\right) .
\end{aligned}
$$

We can solve for the constants, $A_{1}, A_{2}, \phi_{1}$ and $\phi_{2}$ quite easily, but we get rather large expressions,

$$
A_{1}=A \frac{\sqrt{\left(p u-\gamma-p m \omega^{2}\right)^{2}+\nu^{2} \omega^{2}(u-\gamma)^{2}}}{\Delta}
$$

and

$$
A_{2}=A \frac{\sqrt{\left(\xi-\gamma-m k^{2} \omega^{2}\right)^{2}+\nu^{2} \omega^{2}(\xi-p \gamma)^{2}}}{\Delta},
$$

where,

$$
\begin{gathered}
\Delta^{2}=\left(\xi u-m \xi \omega^{2}-m u k^{2} \omega^{2}+m^{2} k^{2} \omega^{2}-\xi \nu u \omega^{2}+\right. \\
\left.\gamma^{2} \nu^{2} \omega^{2}-\gamma^{2}\right)^{2}+\left(\xi \nu u \omega-m k^{2} \nu u \omega^{3}+\right. \\
\left.\xi u \omega-m \xi \omega^{3}-2 \nu \gamma^{2} \omega\right)^{2} .
\end{gathered}
$$

The phase difference is given by $\tan \left(\phi_{2}-\phi_{1}\right)=Y / X$, with,

$$
\begin{array}{cc}
Y= & \left(p u-\gamma-p m \omega^{2}\right)\left(\xi-\gamma-m k^{2} \omega^{2}\right)+ \\
\nu^{2} \omega^{2}(u-\gamma)(\xi-p \gamma) \\
X= & \left(p u-\gamma-p m \omega^{2}\right)(\xi-p \gamma) \nu \omega- \\
& \left(\xi-\gamma-m k^{2} \omega^{2}\right)(u-\gamma) \nu \omega .
\end{array}
$$


Finally here notice that the constants defined above satisfy the following relation,

$$
X^{2}+Y^{2}=\left(\frac{A_{1} \Delta}{A}\right)^{2}\left(\frac{A_{2} \Delta}{A}\right)^{2} .
$$

This is simple to see from the complex arithmetic used to solve the dynamic equations, but it could also be verified directly.

\section{MOTION OF THE BOwL}

Consider a point on the helical track with initial coordinates $\mathbf{q}=(R, 0,0)^{T}$ say, in this linear approximation we can use equation (2) to find the motion of this point:

$$
\mathbf{q}(t)=\left(\begin{array}{c}
R \\
R A_{1} \cos \left(\omega t+\phi_{1}\right) \\
A_{2} \cos \left(\omega t+\phi_{2}\right)
\end{array}\right) .
$$

This is a small, elliptical motion, see Fig. 3.

The 'tilt' and size of the ellipse will be determined by the mechanical parameters of the machine and the frequency of the driving wrench. Notice that the points on the ellipse will satisfy the quadratic equation,

$$
\frac{y^{2}}{A_{1}^{2} R^{2} \sin ^{2} \phi}-\frac{2 y z \cos \phi}{A_{1} A_{2} R \sin ^{2} \phi}+\frac{z^{2}}{A_{2}^{2} \sin ^{2} \phi}=1,
$$

where $\phi=\phi_{2}-\phi_{1}$ is the phase difference. This can be written in matrix form as,

$$
(y, z)\left(\begin{array}{cc}
\frac{1}{A_{1}^{2} R^{2} \sin ^{2} \phi} & -\frac{\cos \phi}{A_{1} A_{2} R \sin ^{2} \phi} \\
-\frac{\cos \phi}{A_{1} A_{2} R \sin ^{2} \phi} & \frac{1}{A_{2}^{2} \sin ^{2} \phi}
\end{array}\right)\left(\begin{array}{l}
y \\
z
\end{array}\right)=1 .
$$

The tilt angle can be found by diagonalising the $2 \times 2$ matrix in equation (45) using a rotation matrix. The tilt angle $\alpha$, will be the rotation angle which diagonalises the coefficient matrix, it satisfies,

$$
\tan (2 \alpha)=\frac{2 A_{1} A_{2} R \cos \phi}{A_{1}^{2} R^{2}-A_{2}^{2}} .
$$

In fact this gives two solutions for $\alpha$, separated by $\pi / 2$, corresponding to the major and minor axes of the ellipse. Notice that this tilt angle is independent of the amplitude of the driving wrench $A$.

We can find the lengths of the semimajor and semiminor axes, $a, b$, by looking at invariants of the matrix. For the diagonalised matrix the determinant will be $1 / a^{2} b^{2}$ and since the determinant is an invariant of the matrix we can evaluate the determinant of the matrix in equation (45) above to give,

$$
a b=A_{1} A_{2} R|\sin \phi| .
$$

The modulus sign here is because $a$ and $b$ are both positive. The trace of the diagonal coefficient matrix is $\left(1 / a^{2}\right)+$ $\left(1 / b^{2}\right)$, this leads to the result,

$$
a^{2}+b^{2}=A_{1}^{2} R^{2}+A_{2}^{2} .
$$

Dividing equation (48) by (47) we get a quadratic equation for the eccentricity of the ellipse $e=a / b$.

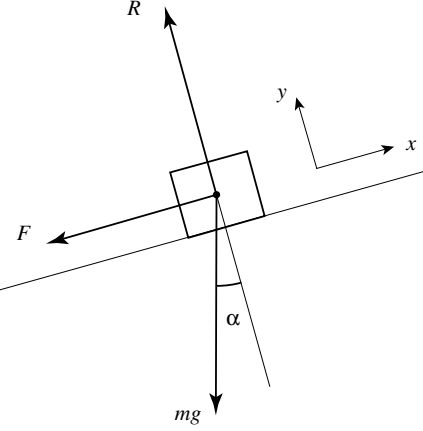

Fig. 4. Force diagram for a part on an inclined plane.

Most literature on bowl feeders state that the device is designed to run at or near resonance. Standard definitions of the resonant frequency roughly state that this is the frequency with maximum output. In our case we have two outputs $\theta_{z}$ and $z$ so we need a slightly modified notion of resonance. Let us assume that we are interested in the situation where the motion of the bowl is maximal. So the "output" of our system can be taken as the area of the ellipse. The area of an ellipse is proportional to the product of its semimajor and semiminor axes $a b$, so we must maximise equation (47). Now we can write $\sin \phi=$ $Y / \sqrt{X^{2}+Y^{2}}$ using $X$ and $Y$ from equations (41) and (40). Then simplifying using equation (42) gives the result,

$$
a b=\frac{A^{2} R|Y|}{\Delta^{2}} .
$$

This expression can be maximised with respect to $\omega$ to yield the condition for resonance. However, in a practical design the driving frequency $\omega$ will be the frequency of the local electricity supply; $2 \pi \times 50 \mathrm{~Hz}$ in Europe and $2 \pi \times 60$ in the US. It is also common to use a rectifier to double this frequency, but in any design study the driving frequency should be considered as fixed and not a design parameter. The resonance condition can be used to optimise a single design parameter the rest being fixed. The easiest to find would be $p$ the pitch of the driving wrench but in most designs this is chosen to be 0 or infinite, where 0 pitch corresponds to a pure force along the axis and infinite pitch corresponds to a pure torque about the axis of the device. It would also be possible to use this condition to optimise with respect to properties of the spring, for example the length of the spring or its angle $\beta$.

\section{PART DYNAMICS}

In [3] it is suggested that the motion of the fed parts in such a vibratory feeder is a bouncing or hopping motion. However, it has also been suggested that the motion is a "slip-stick" motion resulting from the difference between static and dynamic friction. Here we investigate this claim and show that this slip-stick motion is rather unlikely.

Figure 4 shows the forces acting on the fed part when it is in contact with the helical track. In our linear approximation the track is just an inclined plane. For simplicity 
we will assume that the motion of the bowl follows an ellipse with its major axis aligned with the track. In fact, other things being equal, this arrangement will produce the smallest accelerations perpendicular to the track. In coordinates aligned with the inclined plane the motion of the part fixed to the inclined plane will be

$$
\mathbf{q}(t)=\left(\begin{array}{c}
a \cos \omega t \\
b \sin \omega t
\end{array}\right) \text {. }
$$

Applying Newton's second law to the motion perpendicular to the plane gives

$$
R=m_{0} g \cos \alpha-b \omega^{2} \sin \omega t,
$$

where $m_{0}$ is the mass of the part and $g$ is the acceleration due to gravity. (Note that in Fig. $4 F$ represents the frictional force acting on the part.) For the part to stay in contact with the plane the reaction force $R$ must remain positive, implying that,

$$
b \sin \omega t<\frac{m_{0} g}{\omega^{2}} \cos \alpha .
$$

The constant $b$ is positive so we consider the maximum values of $\sin \omega t$, this leads to the inequality,

$$
b<\frac{m_{0} g}{\omega^{2}} \cos \alpha .
$$

Now if we assume that the driving frequency is $50 \mathrm{~Hz}$ and the mass of the part is a few grams, then we see that for no bouncing the displacement of the track perpendicular to its surface must be less than a few microns. This very stringent limit is unlikely to be maintained under normal factory conditions.

\section{CONCLUSIONS}

In this paper we have laid the foundations for an analysis of vibratory bowl feeders. These are important practical machines and it is perhaps a little surprising that the existing literature concerning these devices is so sparse.

Specifically we have constructed a simple linear model for the dynamics of the bowl supported by a symmetrical arrangement of springs. With this simple model we are able to deduce a condition for resonance in terms of the design parameters of the device and the driving frequency of the actuators. We are also able to show, from a theoretical perspective, that the probable mechanism for the motion of the parts is not "slip-stick".

In any modelling exercise it is necessary to make reasonable assumptions and ignore some features of the system. Here for example, we have ignored the fact that most of these machines rest on rubber feet for vibration isolation. We have also approximated the motion of the bowl using linear dynamics. To justify these assumptions we really need to appeal to experiments. Preliminary results show that the motion of the bowl is indeed elliptical but at the moment we do not have any quantative results. However, the modelling techniques we have used are robust and we do not anticipate any major difference between this theory and any experiment. Rather we expect to be able to use suitable experiments to identify some of the model parameters which are difficult to predict from simple measurements, such as the damping factor $\nu$ and the radius of gyration $k$.

For brevity here, we have also ignored the dynamics of the bowl away from the $z$-axis. That is we have tacitly assumed that motions which are neither rotations about the $z$-axis nor translations along it, are fully damped. However, deviations from symmetry, which will always be present to some extent in a real device, may cause energy to be transferred to these "off-axis" modes. In [6] it was suggested that motion in these modes could be the cause of observed "dead-spots" in real devices.

To carry this theoretical work forward we need to study the bouncing dynamics alluded to in [3]. In particular we would like to know how the tilt angle and eccentricity of the elliptical motion of the bowl effects the motion of the parts. Also the pitch of the helical track which carries the parts around the inside of the bowl, is an important design parameter and its effect of the motion of the parts needs to be understood.

We would like to be able to explain certain phenomena observed by operators of these machines. For example, in some cases one can increase the feed rate of the parts by reducing the amplitude of the drive. Also, in some operating regimes the parts will move down the track rather than up. Eventually it should be possible to optimise the design of these machines and hence make them more efficient and more productive.

\section{REFERENCES}

[1] G.M.L. Gladwell and W.M. Mansour. Simulation of vibratory feeders. Proceedings of the symposium on computer-aided engineering. University of Waterloo, pages 215-249, 1971.

[2] E.D Fasse and P.C. Breedveld. Modeling of Elastically Coupled Bodies: Part 1-General theory and Geometric Potential function Method. ASME J. of Dynamical Systems, Measurement and Control, 120 pp. 2544-2550, 1998.

[3] W.H. Huang and M. Mason. Mechanics for vibratory manipulation. Proceedings of the 1997 IEEE International Conference on Robotics and Automation, Albuquerque, NM.

[4] W.M. Mansour. Analog and digital analysis and synthesis of oscillatory tracks. Transactions of the ASME - Journal of Engineering for Industry, 94(2):488-494,1972.

[5] G.P. Maul and M.B. Thomas. A systems model and simulation of the vibratory bowl feeder. Journal of Manufacturing Science, 16(5):309314, 1997.

[6] D. Morrey and J.E. Mottershead. Modelling of vibratory bowl feeders. Proc. I. Mech. E., 200(C6):431-437, 1986.

[7] J.M. Selig. Geometric Fundamentals of Robotics. Springer Verlag, New York, 2005.

[8] J.M. Selig. The spatial stiffness matrix from simple stretched springs. In IEEE Conference on Robotics and Automation, San Francisco, pages 3314-3319. IEEE, 2000.

[9] R. Silversides, J.S. Dai, L. Seneviratne. Force Analysis of a Vibratory Bowl Feeder for Automatic Assembly, to appear in ASME transaction, Journal of Mechanical Design, 207(1), 2005.

[10] A.F. van der Stappen, R.-P. Berretty, K. Goldberg, and M.H. Overmars, Geometry and Part Feeding, in: Modelling of Sensor-Based Intelligent Robot Systems, (H. Bunke, H.I. Christensen, G. Hager, R. Klein Eds.) Lecture Notes in Computer Science, Springer Verlag, Berlin (2001). To appear.

[11] R. von Mises. Motorrechnung, ein neues Hilfsmittel in der Mechanik, Zeitschrift für Angewandte Mathematik und Mechanik Band 4, Heft 2, S.155-181, 1924. English Translation by E.J. Baker and K. Wohlhart. Motor Calculus, a New Theoretical Device for Mechanics. Institute for Mechanics, University of Technology Graz, Austria, 1996. 